\title{
The field high-amplitude SX Phoenicis variable BL Camelopardalis: results from a multisite photometric campaign
}

\section{Pulsation}

\author{
E. Rodríguez ${ }^{1}$, S. Fauvaud ${ }^{2,3}$, J. A. Farrell ${ }^{4}$, A.-Y. Zhou ${ }^{5}$, J.-P. Sareyan ${ }^{6}$, M. J. López-González ${ }^{1}$, M.-A. Dupret ${ }^{7}$, \\ A. Grigahcéne ${ }^{8}$, J. de Ridder ${ }^{9}$, G. Klingenberg ${ }^{10}$, M. Wolf ${ }^{11}$, P. Van Cauteren ${ }^{12}$, P. Lampens ${ }^{13}$, D. Martínez ${ }^{1}$, \\ V. M. Casanova ${ }^{1}$, F. J. Aceituno ${ }^{1}$, M. Helvaci ${ }^{14}$, E. G. Hintz ${ }^{15}$, L. Král ${ }^{16}$, F. Fumagalli ${ }^{3}$, J. H. Simonetti ${ }^{17}$, \\ B. H. Granslo ${ }^{10}$, L. Kotková ${ }^{18}$, G. Santacana ${ }^{2}$, J. Michelet ${ }^{19}$, M. Fauvaud ${ }^{2}$, J.-M. Vugnon ${ }^{20}$, H. Sadsaoud ${ }^{8}$, \\ S. Aliouane ${ }^{8}$, Z. C. Grigahcéne ${ }^{8}$, S. Martín-Ruíz ${ }^{1}$, P. J. Amado ${ }^{1}$, J. C. Suárez ${ }^{1}$, A. Moya ${ }^{1}$, H. Kučáková ${ }^{16}$, R. Kocián ${ }^{16}$, \\ K. Truparováa ${ }^{16}$, A. Avdibegović1 ${ }^{11}$, M. Blažek ${ }^{11}$, J. Kliner ${ }^{11}$, P. Zasche ${ }^{11}$, M. Vilásek ${ }^{16}$, S. Bartošíková ${ }^{16}$, \\ B. Yasarsoy ${ }^{21}$, C. Ulusoy ${ }^{21}$, K. A. Graham ${ }^{22}$, M. P. Nicholson ${ }^{22}$, O. Trondal ${ }^{10}$, C. Yesilyaprak ${ }^{23}$, and D. Coker ${ }^{14}$
}

(Affiliations can be found after the references)

Received 20 March 2007 / Accepted 7 June 2007

\section{ABSTRACT}

Context. BL Cam is an extreme metal-deficient field high-amplitude SX Phe-type variable where a very complex frequency spectrum is detected, with a number of independent nonradial modes excited, unusual among the high-amplitude pulsators in the Lower Classical Instability Strip. Aims. An extensive and detailed study has been carried out to investigate the pulsational content and properties of this object.

Methods. The analysis is based on $283 \mathrm{~h}$ of CCD observations obtained in the Johnson $V$ filter, during a long multisite photometric campaign carried out along the Northern autumn-winter of 2005-2006. Additionally, multicolour BI photometry was also collected to study the phase shifts and amplitude ratios, between light curves obtained in different filters, for modal discrimination of the main excited modes.

Results. The detailed frequency analysis revealed a very rich and dense pulsational content consisting of 25 significant peaks, 22 of them corresponding to independent modes: one is the already known main periodicity $f_{0}=25.5765 \mathrm{~cd}^{-1}(\Delta V=153 \mathrm{mmag})$ and the other 21 are excited modes showing very small amplitudes. Some additional periodicities are probably still remaining in the residuals. This represents the most complex spectrum ever detected in a high-amplitude pulsator of this type. The majority of the secondary modes suspected from earlier works are confirmed here and, additionally, a large number of new peaks are detected. The amplitude of the main periodicity $f_{0}$ seems to be stable during decades, but the majority of the secondary modes show strong amplitude changes from one epoch to another. The suspected fundamental radial nature of the main periodicity of BL Cam is confirmed, while the secondary peak $f_{1}=25.2523 \mathrm{~cd}^{-1}$ is identified as a nonradial mixed mode $g_{4}$ with $\ell=1$. The radial double-mode nature, claimed by some authors for the main two frequencies of BL Cam, is not confirmed. Nevertheless, the frequency $f_{6}=32.6464 \mathrm{~cd}^{-1}$ could correspond to the first radial overtone.

Key words. stars: variables: $\delta$ Sct - stars: individual: BL Camelopardalis - stars: oscillations - techniques: photometric

\section{Introduction}

High-amplitude pulsators in the Lower Classical Instability Strip (that is, $\delta$ Sct (Pop. I) and SX Phe (Pop. II) variables with full visual amplitudes larger than roughly 0.3 ) have been assumed for a long time to present very simple spectra with only one or two radial modes excited, mainly the first two radial modes. However, detailed studies, carried out during recent years, have shown a few exceptions to this general picture. The most remarkable one is that of the high-amplitude $\delta$ Sct star V974 Oph $(\Delta V=$ 0.4, Rodríguez et al. 2000) where Poretti (2003) finds five independent frequencies, with several likely nonradial modes, resembling the much more complex power spectra of the lowamplitude $\delta$ Sct variables. In a few other cases of high-amplitude pulsators, previously assumed as pure monoperiodic (DY Peg) or biperiodic (AI Vel, SX Phe or RV Ari) (Garrido \& Rodríguez 1996; Walraven et al. 1992; Pócs et al. 2002), one or more additional radial and/or nonradial excited modes have been detected with very small amplitudes. Hence, these objects can be considered as very interesting targets for asteroseismological studies.
BL Cam is probably the best of these targets, at least the most complex. This is a field high-amplitude SX Phe variable $\left(\Delta V=0^{\mathrm{m}} 33\right.$, Rodríguez et al. 2000) with very low metal content $([\mathrm{M} / \mathrm{H}]=-2.4$, McNamara 1997) where multiperiodicity has been detected in its light curves by different authors (see Fauvaud et al. 2006, for a review, hereafter F06). Besides the main periodicity, at least six additional independent modes seem to be excited in this star, the majority probably nonradial. The possibility of a radial double-mode nature for the main two periodicities has also been claimed by some authors, similar to a typical "pure" radial double-mode high-amplitude $\delta$ Sct and SX Phe-type variables. However, in this double-mode hypothesis, the true value of the secondary frequency around $31.6 \mathrm{~cd}^{-1}$, or its $1 \mathrm{~cd}^{-1}$ alias $32.6 \mathrm{~cd}^{-1}$, need to be further investigated. This determination is very important as different frequency ratios have consequences in understanding its double-mode nature in the Petersen diagrams (Poretti et al. 2005; Suárez et al. 2006).

Concerning the behaviour of the main periodicity in the (O-C) diagram, F06 found that this can be described by two terms: 1) a secular increase of the main periodicity at a rate of 
Table 1. Participating sites and instrumentation. Observatory code: $\mathrm{AlO}=$ Algiers Observatory, $\mathrm{Bak}=\mathrm{Baker}$ Observatory, $\mathrm{BHO}=\mathrm{Beersel}$ Hills Observatory, $\mathrm{Bos}=$ Bossmo Observatory, $\mathrm{Cal}=$ Calina Observatory, $\mathrm{ChR}=$ Château-Renard Observatory, DAO $=$ Dominion Astrophysical Observatory, Ege = Ege University Observatory, $\mathrm{FjO}=$ Fjellhamar Observatory, Gil = Gilman Observatory, GOO=Groruddalen Optical Observatory, $\mathrm{Mah}=$ Mahill Observatory, Mar = Martin Observatory, OJP = Observatory and Planetarium of Johann Palisa, Ond = Ondrejov Observatory, $\mathrm{OPO}=$ Orson Pratt Observatory at Brigham Young University, PiM = Pic du Midi Observatory, SFO= Sulphur Flats Observatory, $\mathrm{SNO}=$ Sierra Nevada Observatory, TNO = Turkish National Observatory. Notes: a) filter $V$ was used on 2 nights, but filter $B$ only one night, b) filters $I$ and $R$ were also used on 2 and 1 nights, respectively, c) 7 nights in filter $V$ and 5 using filter $R$, d) 20 nights without filter, 4 using filter $R$ and one using Johnson $V$ filter, e) 5 nights with a Bessel $V$ filter and the last 3 ones without filter, f) filter $B$ was not used during the first observing night of this run. The number of hours collected in $V, B$ and $I$ filters was 76.7, 64.0 and 72.0, respectively, g) one observing night in filter $V$ and another night using both $B$ and $V$ filters.

\begin{tabular}{|c|c|c|c|c|c|c|c|c|}
\hline Obs & Location & Longitude & Latitude & $\begin{array}{c}\text { Telescope } \\
\text { (m) }\end{array}$ & Filters & Nights & Hours & $\overline{\text { Notes }}$ \\
\hline TNO & Antalya (Turkey) & $30^{\circ} 20^{\prime} \mathrm{E}$ & $36^{\circ} 49^{\prime} \mathrm{N}$ & 1.50 & $\overline{B V}$ & 2 & 13.1 & $\mathrm{a}$ \\
\hline Ege & Izmir (Turkey) & $27^{\circ} 16^{\prime} \mathrm{E}$ & $38^{\circ} 25^{\prime} \mathrm{N}$ & 0.40 & V & 1 & 4.6 & \\
\hline OJP & Ostrava (Czech Republic) & $18^{\circ} 09^{\prime} \mathrm{E}$ & $49^{\circ} 50^{\prime} \mathrm{N}$ & 0.20 & $V$ & 11 & 34.1 & $\mathrm{~b}$ \\
\hline Ond & Ondrejov (Czech Republic) & $14^{\circ} 47^{\prime} \mathrm{E}$ & $49^{\circ} 55^{\prime} \mathrm{N}$ & 0.65 & $V R$ & 12 & 30.0 & $\mathrm{c}$ \\
\hline Bos & Mo i Rana (Norway) & $14^{\circ} 07^{\prime} \mathrm{E}$ & $66^{\circ} 20^{\prime} \mathrm{N}$ & $0.20 / 0.25$ & no & 25 & 140.2 & $\mathrm{~d}$ \\
\hline $\mathrm{FiO}$ & Fiellhamar (Norway) & $10^{\circ} 58^{\prime} \mathrm{E}$ & $59^{\circ} 56^{\prime} \mathrm{N}$ & 0.20 & $V$ & 4 & 7.8 & \\
\hline GOO & Groruddalen (Norway) & $10^{\circ} 55^{\prime} \mathrm{E}$ & $59^{\circ} 57^{\prime} \mathrm{N}$ & 0.25 & no & 1 & 1.0 & \\
\hline $\mathrm{Cal}$ & Carona (Switzerland) & $8^{\circ} 56^{\prime} \mathrm{E}$ & $45^{\circ} 47^{\prime} \mathrm{N}$ & 0.30 & no & 5 & 17.1 & \\
\hline $\mathrm{ChR}$ & Saint-Véran (France) & $6^{\circ} 54^{\prime} \mathrm{E}$ & $44^{\circ} 42^{\prime} \mathrm{N}$ & 0.62 & V & 11 & 96.7 & \\
\hline BHO & Beersel (Belgium) & $4^{\circ} 18^{\prime} \mathrm{E}$ & $50^{\circ} 46^{\prime} \mathrm{N}$ & 0.40 & $V$ & 8 & 24.0 & $\mathrm{e}$ \\
\hline $\mathrm{SNO}$ & Granada (Spain) & $3^{\circ} 23^{\prime} \mathrm{E}$ & $37^{\circ} 04^{\prime} \mathrm{N}$ & 1.50 & $B V I$ & 13 & 76.7 & $\mathrm{f}$ \\
\hline $\mathrm{AlO}$ & Algiers (Algeria) & $3^{\circ} 03^{\prime} \mathrm{E}$ & $36^{\circ} 47^{\prime} \mathrm{N}$ & 0.80 & $V$ & 1 & 1.9 & \\
\hline PiM & Pic du Midi (France) & $0^{\circ} 09^{\prime} \mathrm{E}$ & $42^{\circ} 56^{\prime} \mathrm{N}$ & 0.60 & no & 4 & 14.4 & \\
\hline Mar & Blacksburg (USA) & $80^{\circ} 33^{\prime} \mathrm{W}$ & $37^{\circ} 20^{\prime} \mathrm{N}$ & 0.40 & $B V$ & 2 & 4.0 & $\mathrm{~g}$ \\
\hline Gil & Gilman (USA) & $87^{\circ} 56^{\prime} \mathrm{W}$ & $41^{\circ} 27^{\prime} \mathrm{N}$ & 0.30 & $V$ & 2 & 8.4 & \\
\hline Bak & Springfield (USA) & $93^{\circ} 02^{\prime} \mathrm{W}$ & $37^{\circ} 24^{\prime} \mathrm{N}$ & 0.40 & V & 7 & 25.8 & \\
\hline Mah & Mahill (USA) & $105^{\circ} 32^{\prime} \mathrm{W}$ & $32^{\circ} 54^{\prime} \mathrm{N}$ & 0.25 & $V$ & 2 & 5.7 & \\
\hline SFO & Jemez Springs (USA) & $106^{\circ} 38^{\prime} \mathrm{W}$ & $35^{\circ} 53^{\prime} \mathrm{N}$ & 0.41 & $V$ & 9 & 86.7 & \\
\hline $\mathrm{OPO}$ & Provo (USA) & $111^{\circ} 39^{\prime} \mathrm{W}$ & $40^{\circ} 14^{\prime} \mathrm{N}$ & 0.40 & V & 4 & 8.4 & \\
\hline DAO & Victoria (Canada) & $123^{\circ} 25^{\prime} \mathrm{W}$ & $48^{\circ} 31^{\prime} \mathrm{N}$ & 1.80 & V & 3 & 5.3 & \\
\hline
\end{tabular}

$\mathrm{d} P / P \mathrm{~d} t=117( \pm 3) \times 10^{-9} \mathrm{yr}^{-1}$ and 2) a perturbation from a companion in a binary system with a rather eccentric orbit with period of $10.5( \pm 0.2)$ yr. However, discontinuous variations in the period change could not be completely discarded and a more detailed analysis is needed with the help of additional times of maxima.

For these various reasons, we undertook a follow-up campaign of BL Cam. Our main aim is to perform a detailed, long-term study concerning: 1) its pulsational content and 2) the behaviour of the main period in the $(\mathrm{O}-\mathrm{C})$ diagram. So, we carried out a multisite photometric campaign centred mainly in the period autumn-winter 2005-2006 with observations from a number of observatories in Europe and North America. These observations have been preponderantly collected in the Johnson $V$ filter, in order to perform a detailed frequency analysis. Those obtained in different filters, will be used to find reliable modal discrimination for the main excited modes. The observations considered as additional (i.e. acquired using "smaller" telescopes or "without any filter") will be used to determine additional times of light maxima, in order to further improve the (O-C) analysis (Fauvaud et al. 2007). In the present work we describe the results concerning the star's pulsational content.

\section{Observations}

The data have been collected between August, 2005 and March, 2007 at 20 observatories in Europe and North America. Table 1 presents a summary with the list of the observatories, instrumentation and journal of the observations secured in this work. In total, photometric data have been acquired on
126 nights and $606.7 \mathrm{~h}$, and most of data (i.e. $551.1 \mathrm{~h}$ over 108 nights) have been obtained between August, 2005 and March, 2006. All observations were obtained with CCD cameras. The majority of data were collected using the Johnson $V$ filter in order to get a homogeneous data set to perform a reliable frequency analysis. The integration times, typically 20 to $120 \mathrm{~s}$, have been adopted according to the instrumentation used in each case.

Additionally, some data were obtained in other filters, BRI, depending on the availability of each participating observatory. In particular, at Sierra Nevada Observatory (SNO), the data were acquired sequentially in filters $B V I$ on 13 nights. The integration times were typically of 40,30 and $20 \mathrm{~s}$ in $B, V$ and $I$ filters. Figure 1 shows one example of $B V I$ light curves of BL Cam obtained during one night using the $1.5 \mathrm{~m}$ telescope at SNO. This figure clearly shows the differences in amplitude among different filters and the slight modulation existing along the main periodicity due to additional excited modes.

As seen in Table 1, observations without any filter and/or with some very small telescopes were also acquired. These data were used only to determine new times of light maxima. In total, about 600 new times of light maxima have been obtained in this work, very useful in order to make a detailed study on the $(\mathrm{O}-\mathrm{C})$ diagram and main periodicity behaviour of BL Cam.

In this campaign, unfortunately, there is not any data set from Asia, which could have helped to avoid the $1 \mathrm{~cd}^{-1}$ alias problem entirely (see Table 1). Nevertheless, the power of this alias is strongly reduced to about $56 \%$ thanks to the two continents involved, and to a good choice of the data sets for the subsequent frequency analysis. 


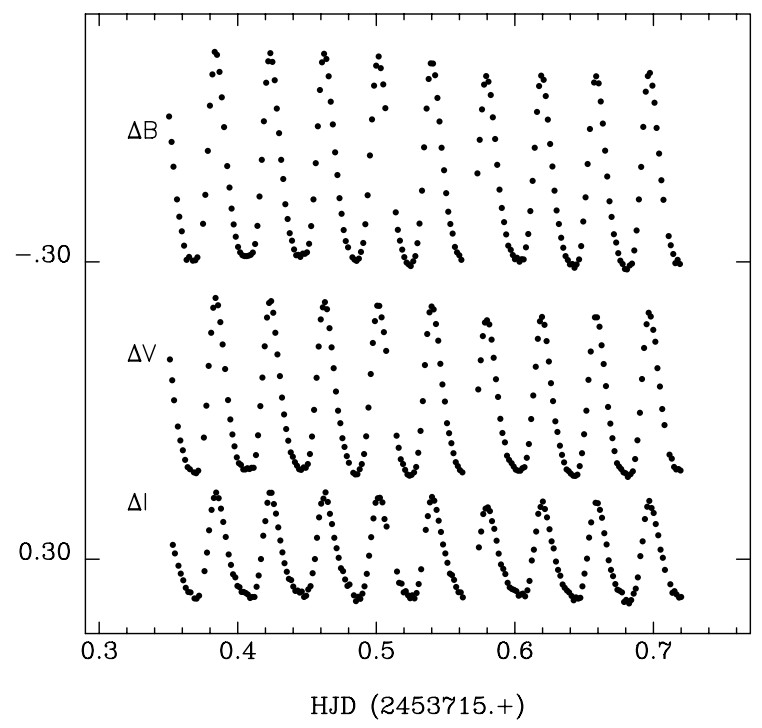

Fig. 1. BVI light curves of BL Cam obtained on December 10th, 2005 at Sierra Nevada Observatory.

\section{Data reduction}

All measurements were obtained by the method of synthetic aperture photometry. Each CCD frame was corrected for dark, bias and flat-field. After checking their stability, stars 4, 9, 12, 15, and 20 (after Fig. 1 of Hintz et al. 1997), located in the same frame as the variable, have been selected as comparison stars for BL Cam in order to derive magnitude differences. The typical photometric uncertainties, estimated from the standard deviation of the magnitude differences between comparison stars, range between 0 . 005 and 0.030 , depending on each participant observatory.

In the particular case of the SNO contribution, typical uncertainties of 5, 6 and 7 mmag were derived in filters $B, V$ and $I$, respectively, for comparison stars of similar magnitude and spectral type than BL Cam. In these frames, more than 20 stars up to about 3 mag fainter than the variable were selected for checking variability. Nevertheless, during the observations reported here, no variations have been found for any of these objects. The upper limit of non-variability depends, in each case, of the apparent magnitude of the stars: from less than one millimagnitude (for those stars of brightness similar to BL Cam) up to nearly 0.01 for the faintest ones. In particular, stars 9 and 12 (Fig. 1 of Hintz et al. 1997), used as main comparison stars for the SNO data reduction, are found as constant above about $0.6 \mathrm{mmag}$ in our region of interest (the region beyond $10 \mathrm{~cd}^{-1}$ ). In the low frequency dominion (below about $2 \mathrm{~cd}^{-1}$ ), this limit increases up to a few millimagnitudes probably due to observational uncertainties.

From our SNO and DAO runs, we confirm the occurrence of an adjacent faint star close to BL Cam, as previously reported in Kim et al. (2003). We estimate its magnitude to be about $V=$ $17 . \mathrm{m}$, and its separation to be $5.1( \pm 0.3)$ arcsec from BL Cam ( $V=13$. 1 , Rodríguez et al. 2000), in agreement with Kim et al. (2003). However, we find a position angle around $256^{\circ}$, according to the definition of such position angle as given by, e.g., Green (1985). This faint star cannot be resolved under poor seeing conditions and/or small size telescopes. Thus, it was not separated in our photometric data reduction from BL Cam itself. Nevertheless, this has not any influence on our results.
Table 2. Contributions used for the frequency analysis. $\sigma$ means residuals after extraction of the full solution of 25 peaks listed in Table 3. Observatory codes are the same as in Table 1.

\begin{tabular}{|c|c|c|c|c|}
\hline Obs & Nights & $\overline{\text { Hours }}$ & $\overline{\text { Points }}$ & $\begin{array}{c}\sigma \\
(\mathrm{mmag})\end{array}$ \\
\hline ChR & 10 & 94.0 & 1435 & 20.8 \\
\hline SNO & 13 & 76.7 & 1719 & 9.1 \\
\hline Bak & 7 & 25.8 & 600 & 11.6 \\
\hline SFO & 9 & 86.7 & 1851 & 9.8 \\
\hline
\end{tabular}

\section{Frequency analysis}

The study of the pulsational content in BL Cam has been carried out following the procedure described in Rodríguez et al. (1998) where Fourier and least-squares algorithms are combined in the same computation program package. This method is similar to that followed by the computation package PERIOD (PERIOD04) (Lenz \& Breger 2005) and both produced identical results in our analysis. When a new peak is found as significant in the frequency domain, this and all the previously determined frequencies are simultaneously optimized and extracted from the data together with the corresponding amplitudes and phases that minimize the residuals. Thus, the method does not depend on successive prewhitening of the data.

We stop the analysis when the new peaks suggested in the periodograms are not formally significant. Following Breger (1993, 1999), a peak is considered as significant when the amplitude signal/noise $(\mathrm{S} / \mathrm{N})$ ratio is larger than 4.0 for independent frequencies or 3.5 for harmonics or frequency combinations. Once all the significant peaks were removed from the periodograms, the noise level was calculated on the residuals. This was made, in a first way, by averaging the amplitudes of the residuals over $5 \mathrm{~cd}^{-1}$ boxes around each frequency under consideration. However, as we will comment later, a mean noise level was also determined over a much larger region of interest.

In order to make our analysis as consistent as possible, only the most reliable contributions from Table 1, with observations in the filter $V$ and longest data sets, were taken into account in our frequency analysis. In this way, we chose the four sets listed in Table 2: two from Europe (ChR and SNO) and another two from America (Bak and SFO). This strongly reduces the power of the daily $\left(1 \mathrm{~cd}^{-1}\right)$ alias from $81 \%$ (when only the SNO contribution is considered) to about $56 \%$ and allows us a better identification of the true peaks in the periodograms, as can be seen in Fig. 2. The full data set considered in Table 2 consists of 5605 measurements collected along $283.2 \mathrm{~h}$ of observations on 39 nights with a time span of 128 days from October, 2005 to March 2006. One night was removed from the global ChR contribution because the corresponding observing run is too short and far from the rest of the nights. The last column in Table 2 is the residual's rms in each contribution after extracting the final solution listed in Table 3. As shown, the ChR data set is much noisier than the others. Thus, this large contribution was considered with weights reduced in about $30 \%$ relative to the rest.

Before performing the frequency analysis on the full data set of Table 2, a sinusoidal variation of amplitude about 0.003 and period about 150 days was detected in the $(\mathrm{O}-\mathrm{C})$ diagram, produced by the light-time effect in a binary or multiple system (Fauvaud et al. 2007). This effect leads to a slight shift of the main frequency, $f_{0}$, depending on the chosen data set. This is probably the cause, at least partially, why different authors find slightly different values for the main frequency $f_{0}$ of BL Cam. A clear example is shown in Table 2 of Zhou et al. (1999). Thus, 


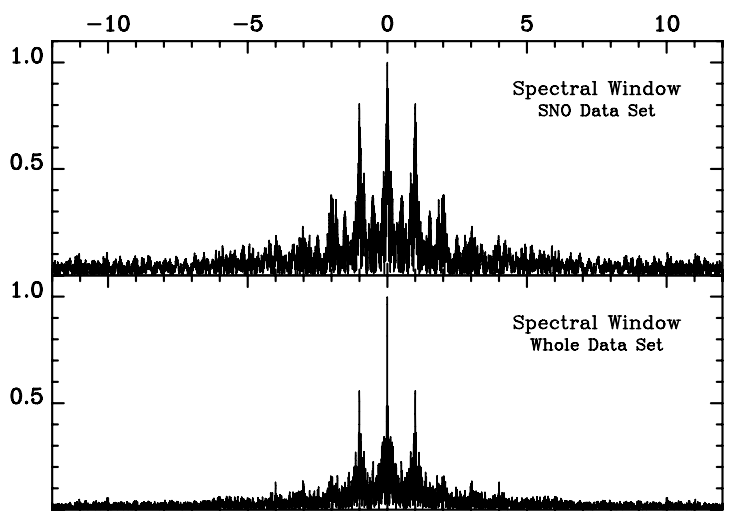

Frequency $(c / d)$

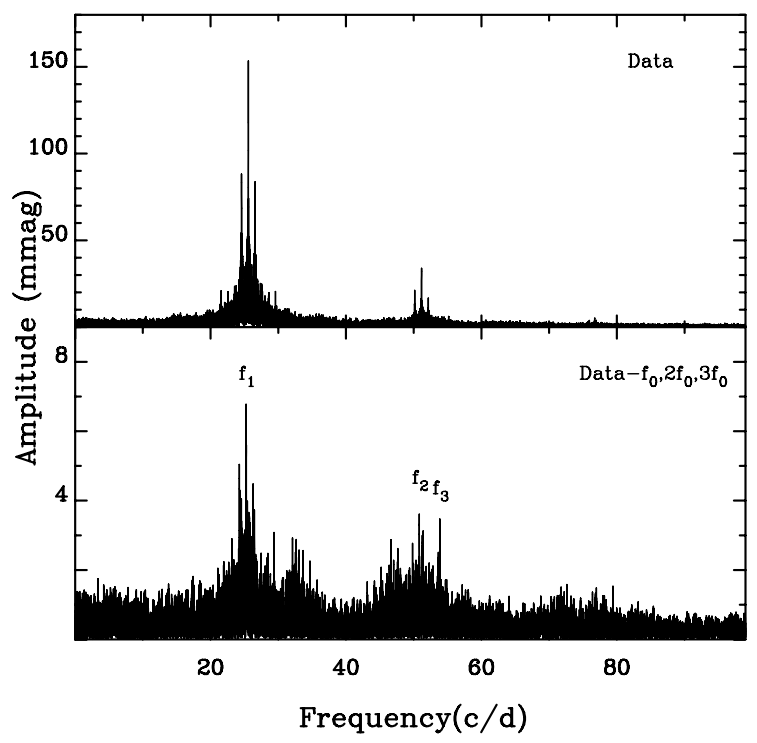

Fig. 2. Top panels: spectral windows of BL Cam in the filter $V$ corresponding to the SNO and whole data sets. Bottom panels: amplitude spectra before and after removing the main periodicity $f_{0}=$ $25.57647 \mathrm{~cd}^{-1}$ together with its two harmonics $2 f_{0}$ and $3 f_{0}$.

we removed this effect from our data before to carry out the frequency analysis.

Moreover, each individual contribution in Table 2 was investigated to avoid misinterpretations in our final results as due to errors coming from a particular subset. This was done with the aim to obtain a final data set as homogeneous as possible. This way, inaccurate points were eliminated, and each data subset was moved to $\langle V\rangle=0$. Next, the full amplitude of the main frequency $f_{0}$ was determined by simultaneously extracting the three significant terms $f_{0}, 2 f_{0}$ and $3 f_{0}$ from each data subset and then compared with that derived from the SNO contribution. As expected, the transformation factors are very close to unity, ranging between 1.028 and 1.065 , i.e. showing a good agreement between the different instrumental systems. Finally, we transformed the instrumental amplitude of each contribution to $\Delta V$ amplitudes of the SNO data set.

\subsection{Results}

A frequency analysis of the full data set revealed the main frequency $f_{0}$ as $25.57647( \pm 0.00001) \mathrm{cd}^{-1}$ with its first two harmonics $2 f_{0}$ and $3 f_{0}$ being significant. Then, the three terms were

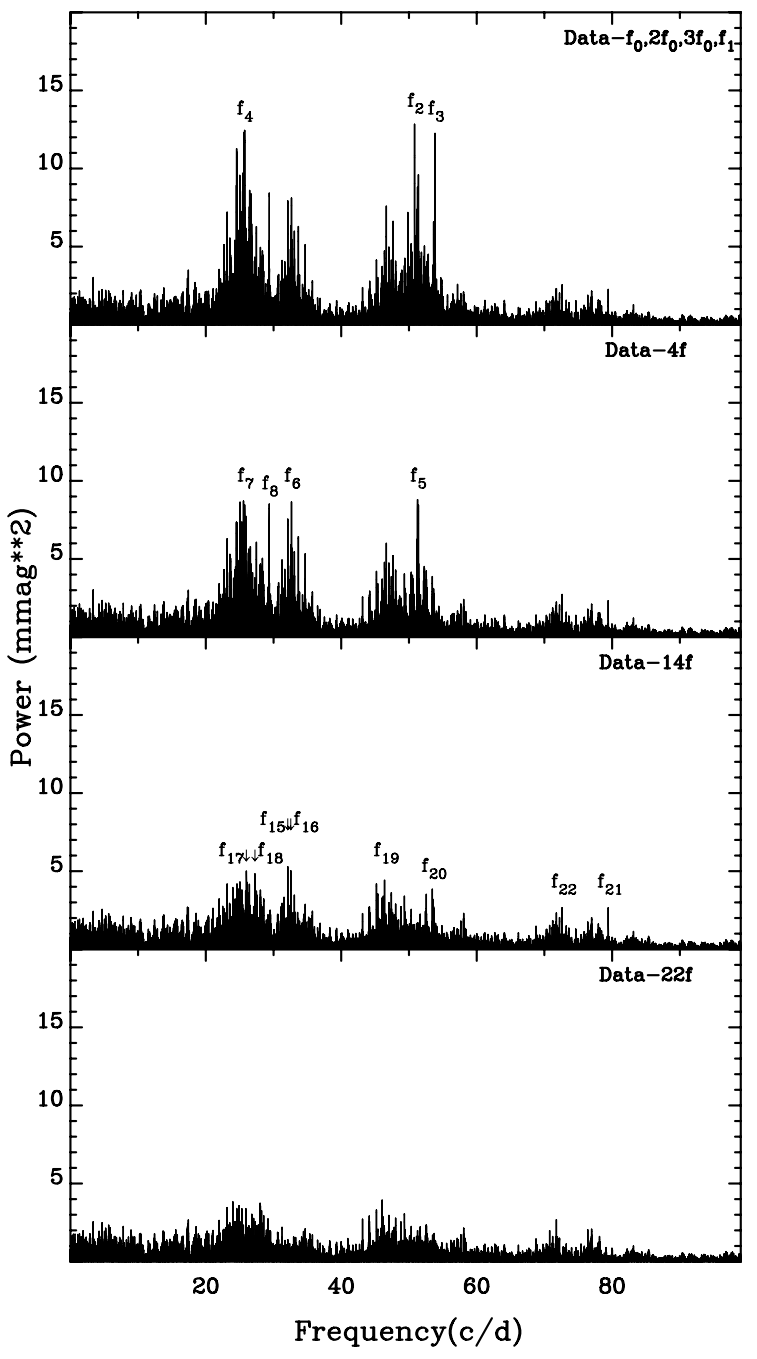

Fig. 3. Power spectra of the residuals of BL Cam after removing different sets of simultaneously optimized peaks.

removed from the data and the residuals were analysed. The results are shown in Figs. 2 and 3 and Table 3. In a first step the periodograms show a main secondary peak $f_{1}=25.25226 \mathrm{~cd}^{-1}$ together with $f_{2}=50.82930 \mathrm{~cd}^{-1}$ and $f_{3}=53.85668 \mathrm{~cd}^{-1} \cdot f_{1}$ was already detected by F06 when analysing the two data sets collected by Zhou et al. $(1999,2001)$, whereas $f_{2}$ corresponds to the coupling term $f_{0}+f_{1}$.

As an additional test, the partial contributions were also checked before analysing the full data set of the residuals in order to prove that, at least, the strongest secondary peaks are intrinsic, not a consequence of artificial coupling of the data. In this sense, the periodograms were investigated in all the $0-f_{N}$ range where $f_{N}$ is the frequency of Nyquist. In our case, $f_{N} \approx 240 \mathrm{~cd}^{-1}$. However, no signal of periodicity was found in the frequency region larger than $80 \mathrm{~cd}^{-1}$.

The situation was quite different in the low frequency domain, i.e. lower than about $5 \mathrm{~cd}^{-1}$ : a number of peaks appeared in this zone (mainly below $2 \mathrm{~cd}^{-1}$ ) with amplitudes ranging from 5 to $2 \mathrm{mmag}$. We consider that none of them is real because in all cases they strongly depend of the data subset considered. Moreover, none of these peaks corresponds to combinations of the high frequency significant peaks. They are probably generated by systematic observational errors as instrumental drifts, residual sky transparency variations or nightly zero-point shifts 
Table 3. Results from Fourier analysis with frequencies ordered in the same order as they appear in the periodograms. Sigma of residuals is $14.2 \mathrm{mmag}$. Signal/noise $S / N$ (1) ratio means that the noise levels are determined using $5 \mathrm{~cd}^{-1}$ boxes around each frequency. $S / N(2)$ means that a mean noise level of $0.49 \mathrm{mmag}$, determined from the intervals 10-20 and 30-45 $\mathrm{cd}^{-1}$, has been used for peaks in the range lower than $60 \mathrm{~cd}$. Notes: a) this peak corresponds to the frequency $f_{2}$ of F06, b) $f_{2}=f_{0}+f_{1}$ and appears in F06 too, c) this peak corresponds to $f_{7}$ in F06, d) this peak corresponds to the $1 \mathrm{~cd}^{-1}$ alias of $f_{1}$ in F06, e) this peak corresponds to the $1 \mathrm{~cd}^{-1}$ alias of $f_{6}$ in F06, f) this peak corresponds to $f_{4}$ in F06.

\begin{tabular}{|c|c|c|c|c|c|c|c|c|c|}
\hline $\begin{array}{l}\text { Frequency } \\
\left(\mathrm{cd}^{-1}\right)\end{array}$ & $\begin{array}{c}\Delta V \\
(\mathrm{mmag})\end{array}$ & $\begin{array}{r}S / N \\
(1)\end{array}$ & $\begin{array}{r}S / N \\
(2)\end{array}$ & Notes & $\begin{array}{c}\text { Frequency } \\
\left(\mathrm{cd}^{-1}\right)\end{array}$ & $\begin{array}{c}\Delta V \\
(\mathrm{mmag})\end{array}$ & $\begin{array}{r}S / N \\
(1)\end{array}$ & $\begin{array}{r}S / N \\
(2)\end{array}$ & $\overline{\text { Notes }}$ \\
\hline & \pm 0.29 & & & & & \pm 0.29 & & & \\
\hline$f_{0}=25.57647$ & 153.29 & 223.2 & 312.8 & & $f_{11}=46.64909$ & 2.60 & 4.3 & 5.3 & \\
\hline $2 f_{0}$ & 34.08 & 61.4 & 69.6 & & $f_{12}=24.54734$ & 3.08 & 4.5 & 6.3 & \\
\hline $3 f_{0}$ & 5.78 & 13.9 & 13.9 & & $f_{13}=51.39570$ & 2.94 & 5.3 & 6.0 & \\
\hline$f_{1}=25.25226$ & 7.35 & 10.7 & 15.0 & $\mathrm{a}$ & $f_{14}=51.18964$ & 2.46 & 4.4 & 5.0 & \\
\hline$f_{2}=50.82930$ & 3.39 & 6.1 & 6.9 & b & $f_{15}=32.10294$ & 2.00 & 3.9 & 4.1 & $\mathrm{e}$ \\
\hline$f_{3}=53.85668$ & 4.44 & 9.1 & 9.1 & & $f_{16}=32.54533$ & 2.07 & 4.0 & 4.2 & $\mathrm{f}$ \\
\hline$f_{4}=25.63866$ & 2.33 & 3.4 & 4.8 & $\mathrm{c}$ & $f_{17}=25.99419$ & 2.64 & 3.8 & 5.4 & \\
\hline$f_{5}=51.25561$ & 2.49 & 4.4 & 5.1 & & $f_{18}=27.26231$ & 2.36 & 3.5 & 4.8 & \\
\hline$f_{6}=32.64641$ & 3.07 & 6.0 & 6.3 & d & $f_{19}=46.39001$ & 2.09 & 3.4 & 4.3 & \\
\hline$f_{7}=25.73943$ & 3.86 & 5.6 & 7.9 & & $f_{20}=53.44596$ & 1.94 & 3.9 & 4.0 & \\
\hline$f_{8}=29.35951$ & 3.05 & 5.1 & 6.2 & & $f_{21}=79.43351$ & 1.66 & 4.9 & 4.9 & \\
\hline$f_{9}=25.01079$ & 3.27 & 4.8 & 6.7 & & $f_{22}=72.60920$ & 1.62 & 4.0 & 4.0 & \\
\hline$f_{10}=25.35245$ & 3.48 & 5.0 & 7.1 & & & & & & \\
\hline
\end{tabular}

in data coming from different observatories. This can increase the noise at higher frequencies due to spectral leakage. For this reason, these peaks were removed from the data and, then, the remaining residuals were analysed. The results are shown in the bottom panel of Fig. 2, Fig. 3, and Table 3. In order to check our results, the resulting frequency solution was compared with that obtained without previously filtering the low-frequency peaks. The results are essentially the same.

In Table 3, the frequencies are ordered in the same order that detected in our analysis. Note that this order is not the same as that corresponding to the decreasing amplitude in the final solution, because the fit including several close frequencies produces changes in the amplitudes. This can be an indication that our solution is not perfect, but we feel that in all cases our detections are reliable, independently of the true amplitude of each peak. Examples are the frequencies $f_{2}, f_{4}$ and $f_{5}$ which are clearly shown in the periodograms of Figs. 2 and 3. Moreover, $f_{2}\left(=f_{0}+f_{1}\right)$ and $f_{4}$ were already detected in the work of F06 (as $f_{3}$ and $f_{7}$, respectively). Hence, there is no reason to mistrust their reality.

Concerning the formal significance of the peaks, in some cases the signal/noise (S/N (1)) ratios are below the limit 4.0. This is because: a) the final amplitudes of these peaks are lower than the "original" amplitudes when they were found in the periodograms and b) in some frequency regions, the noise level is higher than the mean, probably because some periodicities are still remaining in the residuals. If we consider a mean noise level determined from a much larger interval ([10-20] and [30-45] $\mathrm{cd}^{-1}$ ), where the noise seems to be flat, all the peaks can be considered as significant.

As shown in Table 3, some of the modes detected were already found in the work of F06. Indeed, the majority (except $f_{5}=25.91 \mathrm{~cd}^{-1}$ ) of the secondary peaks suspected in that work are confirmed in the present analysis. As summary, we find 25 significant peaks, 22 of them corresponding to independent frequencies: one is the main pulsation frequency with high amplitude and the other 21 are modes showing very small amplitudes. Some peaks could still remain in the residuals. The residual light curves of BL Cam (after extracting the main periodicity $f_{0}$ and its two harmonics $2 f_{0}$ and $3 f_{0}$ ) and Fourier fitting solution are shown in Fig. 4. The synthetic light curve fits most of the observational data, except for a few nights where the observations present higher scatter.

\subsection{Discussion}

\subsubsection{The main frequency and amplitude variations}

As in previous works, the main frequency $f_{0}=$ $25.57647( \pm 0.00001) \mathrm{cd}^{-1}$ shows as significant its first two harmonics only, $2 f_{0}$ and $3 f_{0}$, and seems to be stable from a pulsational point of view.

Nevertheless, as already mentioned, some variability of the main periodicity is detected from the $(\mathrm{O}-\mathrm{C})$ diagram of the light curve maxima as produced (F06) by: a) a secular term that could be due to the stellar evolution, and b) probable binarity with a time scale of several years.

Moreover, a periodic variability with time scale of about 150 days and full amplitude of about 0.003 (probable multiplicity) is also found, from the $(\mathrm{O}-\mathrm{C})$ analysis, when we use the new data of the present multisite photometric campaign (Fauvaud et al. 2007). This can produce slightly different results for $f_{0}$ when performing a Fourier analysis. Indeed, our adopted value for $f_{0}$ is slightly different from $25.5769 \mathrm{~cd}^{-1}$ which corresponds to the mean period determined from the $(\mathrm{O}-\mathrm{C})$ analysis in Zhou et al. (1999). This is in agreement with the slightly different results obtained when different combinations of our data subsets are analysed. This can also be responsible for the slightly different frequencies published in the literature for the main pulsation of BL Cam. As an example, see Table 3 of Zhou et al. (1999).

Concerning the amplitude of $f_{0}$, it seems to be stable too. The value found in this work is of 153.29 mmag (Table 3) which is within the range of values determined in Table 3 of F06 and differs in about $4.7 \%$ from the mean value of $146( \pm 10)$ mmag derived by those authors. Note, that the error bars in amplitude in our Table 3 are only the "formal" error bars from the Fourier fitting, as result of the multiple-frequency nonlinear least-squares fit to the data. These are in very good agreement with those error bars obtained using the analytically derived formula of Montgomery \& O'Donoghue (1999). However, our $V$ magnitudes are instrumental magnitudes, not standard ones: as discussed in previous sections, the $V$ amplitudes determined from the different contributions in our Table 2 differ between $2.8 \%$ 


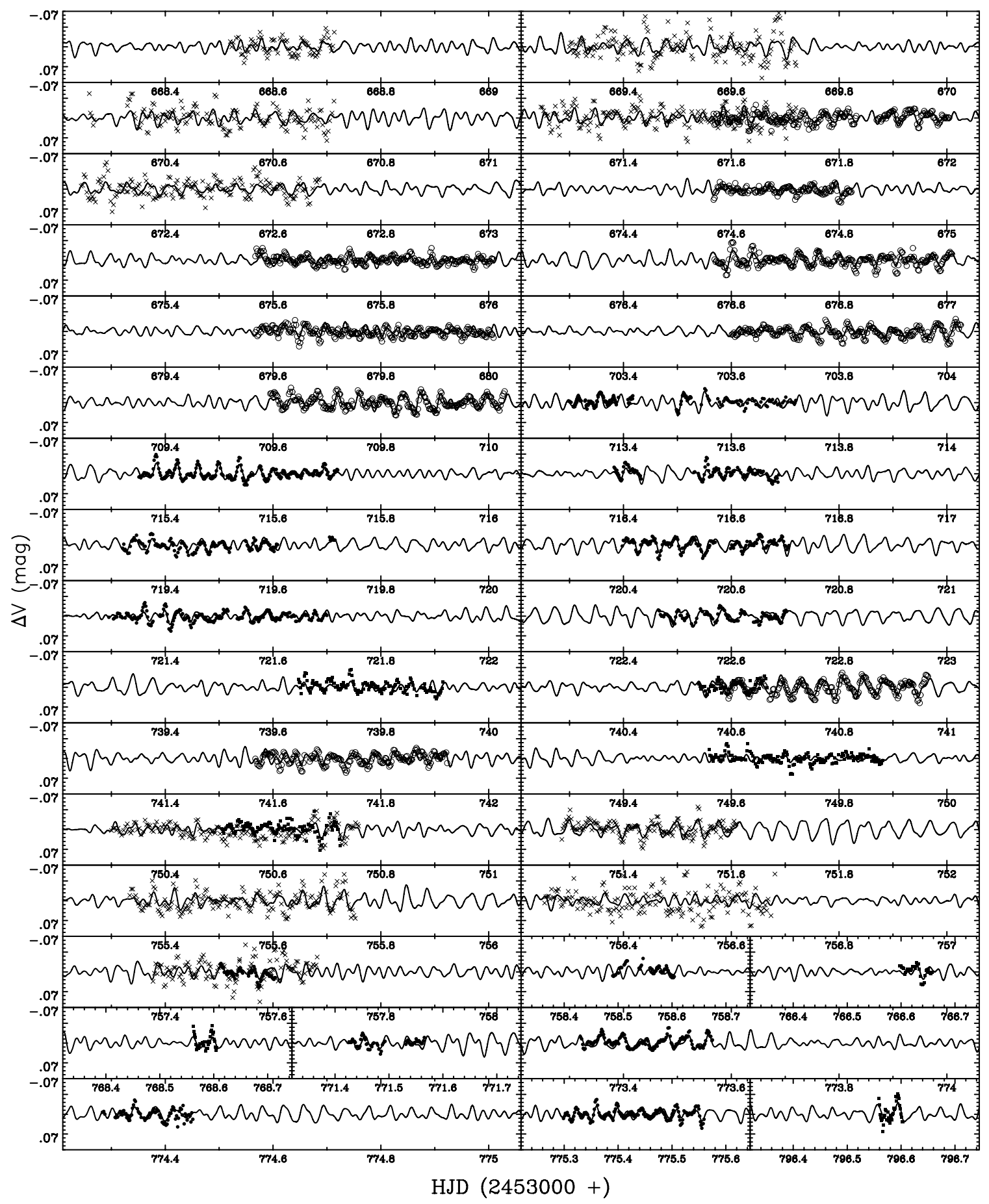

Fig. 4. Light curves of BL Cam corresponding to the residuals after removing the main periodicity and Fourier fitting using the 22 -frequency solution of Table 3. The different contributions are: ChR (crosses), SNO (filled circles), Bak (filled squares) and SFO (open circles).

and $6.5 \%$ from the SNO $V$ amplitude. Thus, we do not find a significant change in the $V$ amplitude of the main term $f_{0}$ of BL Cam when compared to the values found in F06.

Nevertheless, concerning the amplitudes of the secondary modes, the error bars, as due to the use of instrumental magnitudes, are of the same order as the "formal" error bars in Table 3. Thus, we can say that all the secondary modes in Table 3, which were already detected in F06, present changes in amplitude.

Moreover, the small errors mentioned above have no influence in our analysis of the modal discrimination by multicolour photometry that will be carried out in Sect. 5 .

\subsubsection{The $31-32 \mathrm{~cd}^{-1}$ range}

One of the aims of this work was to avoid the $1 \mathrm{~cd}^{-1}$ alias problem and to determine unambiguously if the secondary peak in this zone is around 31.6 or $32.6 \mathrm{~cd}^{-1}$ or if both are present. We also wanted to study the possible double-mode nature of this star, i.e. to see if two radial modes among the main peaks coexist.

Our analysis shows that the true peak exists only in the region around $32.65 \mathrm{~cd}^{-1}$, given as $f_{6}$ in Table 3. Other two detected peaks by F06 in this region are also confirmed in our analysis as $f_{15}=32.10$ and $f_{16}=32.55 \mathrm{~cd}^{-1}$. Hence, detection of multiple peaks in this region is confirmed. 
Table 4. Fourier fit for the main periodicity of BL Cam and the six first frequencies, $f_{1}$ to $f_{6}$, as applied to the three Johnson $B V I$ filters and the data set collected at SNO. Sigmas of the residuals are $15.1(B), 12.0(V)$ and $9.1 \mathrm{mmag}(I)$.

\begin{tabular}{|c|c|c|c|c|c|c|}
\hline & \multicolumn{2}{|c|}{$\bar{B}$} & \multicolumn{2}{|c|}{$\overline{\bar{V}}$} & \multicolumn{2}{|c|}{$\overline{\bar{I}}$} \\
\hline Frequency & $\begin{array}{c}A \\
(\mathrm{mmag})\end{array}$ & $\begin{array}{c}\varphi \\
(\mathrm{rad})\end{array}$ & $\begin{array}{c}A \\
(\mathrm{mmag})\end{array}$ & $\begin{array}{c}\varphi \\
\text { (rad) }\end{array}$ & $\begin{array}{c}A \\
(\mathrm{mmag})\end{array}$ & $\begin{array}{c}\varphi \\
\text { (rad) }\end{array}$ \\
\hline & \pm 0.56 & & \pm 0.42 & & \pm 0.33 & \\
\hline$f_{0}$ & 184.21 & $\begin{array}{r}2.029 \\
4\end{array}$ & 153.74 & $\begin{array}{r}2.015 \\
3\end{array}$ & 91.00 & $\begin{array}{r}1.942 \\
4\end{array}$ \\
\hline $2 f_{0}$ & 43.10 & $\begin{array}{r}6.009 \\
15\end{array}$ & 35.78 & $\begin{array}{r}5.999 \\
13\end{array}$ & 22.14 & $\begin{array}{r}6.012 \\
16\end{array}$ \\
\hline $3 f_{0}$ & 7.58 & $\begin{array}{r}4.304 \\
84\end{array}$ & 5.81 & $\begin{array}{r}4.255 \\
82\end{array}$ & 3.70 & $\begin{array}{r}4.298 \\
95\end{array}$ \\
\hline$f_{1}$ & 8.44 & $\begin{array}{r}1.003 \\
66\end{array}$ & 6.96 & $\begin{array}{r}1.051 \\
61\end{array}$ & 4.30 & $\begin{array}{r}1.081 \\
76\end{array}$ \\
\hline$f_{2}$ & 6.27 & $\begin{array}{r}5.222 \\
90\end{array}$ & 4.89 & $\begin{array}{r}5.185 \\
86\end{array}$ & 2.61 & $\begin{array}{r}5.333 \\
126\end{array}$ \\
\hline$f_{3}$ & 5.30 & $\begin{array}{r}1.686 \\
106\end{array}$ & 4.78 & $\begin{array}{r}1.867 \\
88\end{array}$ & 3.30 & $\begin{array}{r}1.876 \\
100\end{array}$ \\
\hline$f_{4}$ & 4.19 & $\begin{array}{r}3.026 \\
133\end{array}$ & 2.93 & $\begin{array}{r}3.080 \\
143\end{array}$ & 2.11 & $\begin{array}{r}3.162 \\
155\end{array}$ \\
\hline$f_{5}$ & 2.44 & $\begin{array}{r}5.017 \\
230\end{array}$ & 2.44 & $\begin{array}{r}4.886 \\
169\end{array}$ & 1.67 & $\begin{array}{r}5.034 \\
192\end{array}$ \\
\hline$f_{6}$ & 2.86 & $\begin{array}{r}3.336 \\
194\end{array}$ & 2.58 & $\begin{array}{r}3.502 \\
159\end{array}$ & 1.64 & $\begin{array}{r}3.278 \\
193\end{array}$ \\
\hline
\end{tabular}

Table 5. Observed phase shifts and amplitude ratios.

\begin{tabular}{crrrr}
\hline \hline $\begin{array}{c}\text { Frequency } \\
\left(\mathrm{cd}^{-1}\right)\end{array}$ & $\begin{array}{r}B-I \\
\left({ }^{\circ}\right)\end{array}$ & $\begin{array}{r}V-I \\
\left({ }^{\circ}\right)\end{array}$ & $B / I$ & $V / I$ \\
\hline$f_{0}=25.57647$ & 5.0 & 4.2 & 2.02 & 1.69 \\
& 0.4 & 0.4 & 1 & 1 \\
$f_{1}=25.25226$ & -4.4 & -1.7 & 1.96 & 1.62 \\
& 8.1 & 7.8 & 20 & 16 \\
$f_{4}=25.63866$ & -7.8 & -4.7 & 1.99 & 1.39 \\
& 16.5 & 17.1 & 41 & 29 \\
\hline
\end{tabular}

On the other hand, the peak $f_{6}=32.6464 \mathrm{~cd}^{-1}$ implies a period ratio of $f_{0} / f_{6}=0.7834$, very close to the theoretical period ratio corresponding to the first overtone/fundamental radial pulsation modes $(1 \mathrm{H} / \mathrm{F})$ of 0.7843 obtained from the models computed for BL Cam in this work (Sect. 5). It suggests the first radial overtone for $f_{6}$, although the strong amplitude changes which take place in this mode, when compared with previous analyses (see F06), are not a common feature among radial pulsators. Unfortunately, our multicolour photometry cannot be used to discriminate between radial or non-radial nature for this mode because of its actual very small amplitude and, hence, too large uncertainties, as shown in Table 4.

\subsubsection{The $25 \mathrm{~cd}^{-1}$ region}

The majority of the peaks detected in the review by F06 are confirmed by our analysis. The only exception should be that named $f_{5}$ in their work $\left(25.91 \mathrm{~cd}^{-1}\right.$ in Table 6 or $25.94 \mathrm{~cd}^{-1}$ in Table 5). Possibly, failure of detection in our analysis is because its present amplitude is below our photometric detectability level because of the strong amplitude changes that seem to be present in the secondary modes of BL Cam. Nevertheless, this peak might correspond to our detection $f_{17}=25.99 \mathrm{~cd}^{-1}$. On the other hand, some additional peaks have also been detected in the region close to the main frequency which were not found as significant in previous investigations. All these peaks should
Table 6. Main stellar parameters of the theoretical models of BL Cam.

\begin{tabular}{cccccc}
\hline \hline Model & $M\left(M_{\odot}\right)$ & $\log T_{\text {eff }}$ & $\log L / L_{\odot}$ & $\log g$ & $\alpha$ \\
\hline Model 1 & 0.98 & 3.901 & 0.700 & 4.289 & 1.8 \\
Model 2 & 0.98 & 3.901 & 0.699 & 4.289 & 1.0 \\
\hline
\end{tabular}

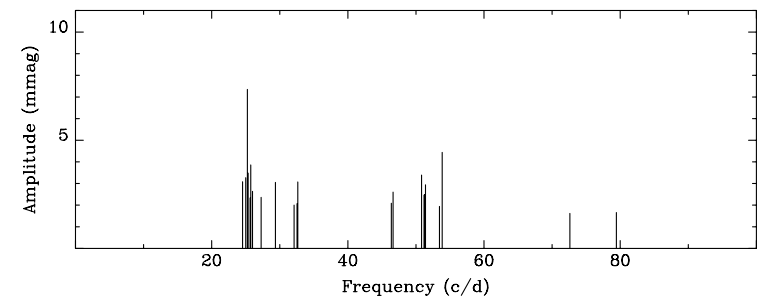

Fig. 5. Distribution of the frequencies of the detected modes. Those corresponding to the main periodicity, $f_{0}, 2 f_{0}$ and $3 f_{0}$, are not shown in the figure.

correspond to nonradial modes if we assume that $f_{0}$ is a radial one, as it will be confirmed in later sections.

Similar to F06, when analysing the best two data sets of BL Cam in the literature (those collected by Zhou et al. 1999, 2001), we also find the secondary peak $f_{1}=25.25226 \mathrm{~cd}^{-1}$ as the one with the highest amplitude. However, as shown in F06, sometimes this is not true. Even, in some epochs this peak was not found as significant. A detailed look to our Table 3 and those from F06 shows that this fact is common in the secondary peaks in the $25 \mathrm{~cd}^{-1}$ region. All these peaks present changes in amplitude from epoch to epoch. Indeed, sometimes some of them are not detected photometrically. As commented before, strong amplitude changes have also taken place in the mode corresponding to $32.6 \mathrm{~cd}^{-1}$. In fact, at some epochs this appears as the highest secondary peak.

\subsubsection{Additional modes and distribution of the peaks}

Figure 5 shows the frequencies detected in this work. This figure clearly shows that the detected peaks are not randomly distributed in all the frequency range, but they tend to cluster in groups as previously found in other multiperiodic $\delta$ Sct pulsators with dense spectrum of frequencies (e.g., Breger et al. 2005).

Besides the secondary peaks in the regions at $25 \mathrm{~cd}^{-1}$ and $32 \mathrm{~cd}^{-1}$, other independent modes have been detected in BL Cam. The majority of them are grouped in well-defined zones of the spectrum: $46,51-53$ and $72-80 \mathrm{~cd}^{-1}$. If we assume that $f_{0}$ is the fundamental pulsational mode $\mathrm{p}_{1}$ (see next section), the peaks in these regions are suggested to correspond to the radial orders $\mathrm{p}_{4}, \mathrm{p}_{5}$ and $\mathrm{p}_{8}-\mathrm{p}_{9}$, respectively. Although this is the first time that these peaks have been found to be significant, the existence of some of them was already suspected in the work of F06. See, for example, the power spectrum of the residuals in Fig. 2 of that work.

One question still remains open: are there also excited modes in the low-frequency domain of the spectrum of BL Cam? The possible peaks in this region could be a consequence of the existence of additional independent modes (in such case, they should correspond to gravity modes) or the result of coupling between the already detected main modes. Unfortunately, due to the high noise present in our periodograms for this frequency region (very probably as consequence of the observational uncertainties commented in Sect. 4.1, difficult to reliably assess at millimagnitude 
level in multiperiodic high-amplitude pulsators), such detection has not been actually possible.

\section{Mode identification}

\subsection{Multicolour observations}

In order to perform a modal identification of the spherical degree $\ell$ for the main excited modes of BL Cam, the multicolour $B V I$ observations collected at SNO were used. Table 4 lists the results of the Fourier fit to the main frequencies until $f_{6}$. The results are consistent in all cases: the peaks are significant in the three filters, the amplitudes decrease as the frequency number increases, for each frequency the relative amplitudes in different filters agree with pulsations, the residuals clearly suggest the existence of additional modes.

Table 5 lists the amplitude ratios and phase shifts obtained for $f_{0}, f_{1}$ and $f_{4}$. A preliminary identification of the degree $\ell$ can be made for $f_{0}$ and $f_{1}$ by comparing this table with Fig. 2 of Balona \& Evers (1999). The main periodicity $f_{0}$ corresponds obviously to a radial mode. In such case $f_{1}$ must be a non-radial mode with $\ell=1$. Concerning $f_{4}$, although we know this must be a non-radial mode (because its frequency ratio relative to $f_{0}$ is close to unity), the error bars are too large to distinguish between $\ell=1$ or $\ell=2$. A more detailed study for the discrimination of these modes is presented below.

\subsection{Models}

We computed different theoretical models of BL Cam with the stellar evolution code CLES (Code Liegeois d'Evolution Stellaire). The global atmospheric stellar parameters of two of these models (different $\alpha$ values) are given in Table 6, in agreement with the parameters $\left(T_{\text {eff }}=7970 \mathrm{~K}, \log g=4.27\right.$ and $[\mathrm{M} / \mathrm{H}]=-2.4)$ derived by McNamara (1997) from photometric and spectroscopic observations carried out for BL Cam. In our models, $X$ and $Z$ are taken as $X=0.76$ and $Z=0.0001$, which are typical for extreme Pop II stars similar to BL Cam. The masses and lumosities in our models are also in very good agreement with those $\left(M=0.99 M_{\odot}\right.$ and $\left.\log L / L_{\odot}=0.692\right)$ determined by McNamara (1997).

We present some evolutionary tracks obtained with the same $X$ and $Z$ and begining at the ZAMS (cf. Fig. 6). We see that for such low $Z$, masses lower than $1 M_{\odot}$ are required to fit our star while crossing the instability strip during the main sequence stage. These Pop II models have no convective core, contrary to Pop I $\delta$ Sct stars. The evolution leading to SX Phe stars is still subject of controversy, and the evolution tracks given in Fig. 6 might not be representative of the real history of BL Cam. But for a given location in the HR diagram, its envelope structure is very similar to standard $\delta$ Sct stars. Hence, the driving of the modes is qualitatively the same (that is, $\kappa$-mechanism in the HeIII partial ionization zone) and the phase lags occur in same regions ( $\mathrm{H}$ and $\mathrm{He}$ partial ionization sones).

The use of the non-adiabatic pulsation code MAD (Dupret 2002), fully tested for $\delta$ Sct stars, is thus entirely justified for the theoretical study of BL Cam. Time-Dependent Convection is included in our non-adiabatic models (Grigahcène et al. 2005), which leads to a much better agreement with observations, as shown by Dupret et al. (2005) for $\delta$ Sct stars. With this code we determined the normalized amplitude and phase of effective temperature variation $f_{T}=\left|\delta T_{\text {eff }} / T_{\text {eff }}\right|$ and $\psi_{T}$, for the models of Table 6 and for modes with different $\ell(0 \leq \ell \leq 3)$ with frequencies closest to the observed values. The values obtained for the

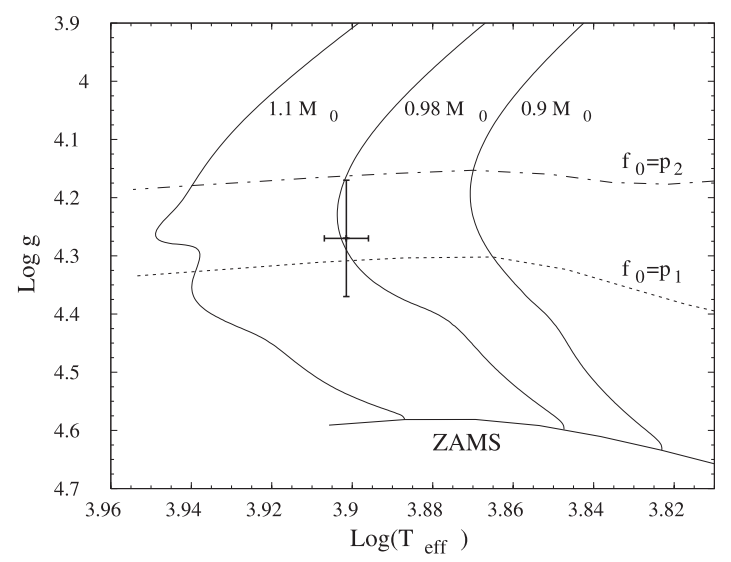

Fig. 6. $\log T_{\text {eff }}-\log g$ diagram with the location of BL Cam deduced from its colour indexes (cross). Some evolutionary tracks $(0.9,0.98$ and $1.1 M_{\odot}$ ) and the location of models fitting exactly $f_{0}$ as the radial fundamental mode (dotted line) or the radial first overtone (dot-dashed line) are also shown.

fundamental radial mode are $f_{T}, \psi_{T}=\left(3.73,111^{\circ}\right)$ for model 1 and $f_{T}, \psi_{T}=\left(4.49,123^{\circ}\right)$ for model 2 . The non-adiabatic results are mainly sensitive to the MLT parameter $\alpha$ as usually found in $\delta$ Sct stars (Moya et al. 2004). We considered models with other $T_{\text {eff }}$ and $\log g$ (inside the observational error bars) and the results obtained are very similar.

Finally, taking these non-adiabatic results as input, we computed the theoretical amplitude ratios and phase differences between different photometric passbands ( $I$ filter taken as reference). To obtain them, we used Kurucz atmosphere models (Kurucz 1993) with $[\mathrm{M} / \mathrm{H}]=-2.5$ and $[\mathrm{M} / \mathrm{H}]=-2.0$ and the limb-darkening law of Claret (2000).

\subsection{Interpretation of the frequencies}

The comparison of our theoretical results with the observations is presented in Fig. 7. It is well known that amplitude ratios are more sensitive to incertitudes in the atmosphere model than phase differences for $\delta$ Scuti stars. Moreover, the phase differences give a better discrimination between different $\ell$. Hence, we are more confident about the phase differences (bottom panels of Fig. 7) for the mode identifications.

As can be seen, the mode $f_{0}$ is identified as a radial mode. The best agreement is found for the model with $\alpha=1$ (left panels, less efficient convection). Comparison with the observed frequency shows that it is probably the radial fundamental mode (see below). The error bars are larger for $f_{1}$. The best discrimination is found in the phase-difference diagram which indicates a $\ell=1$ mode as the most probable. Comparison with observed frequency shows that it would be probably the mixed mode $g_{4}$. Finally, the error bars are too large for $f_{4}$ to allow a reliable mode identification. Because of this frequency being very close to $f_{0}$, it is certainly a non-radial mode.

The degree $\ell$ of the two modes $f_{0}$ and $f_{1}$ are identified. But $m$ is unknown for $f_{1}$. At this stage only the radial mode frequency $f_{0}$ can be used to constrain without arbitrary assumption the model. In Fig. 6, the dotted line corresponds to the location of models fitting exactly $f_{0}$ as the radial fundamental mode $\left(p_{1}\right)$. The dot-dashed line corresponds to models fitting $f_{0}$ as the radial first overtone $\left(p_{2}\right)$. The cross corresponds to the location of BL Cam in the HR diagram as deduced from photometry with typical error bars. We see that the best solution identifies $f_{0}$ as the fundamental radial mode, although the other possibility 

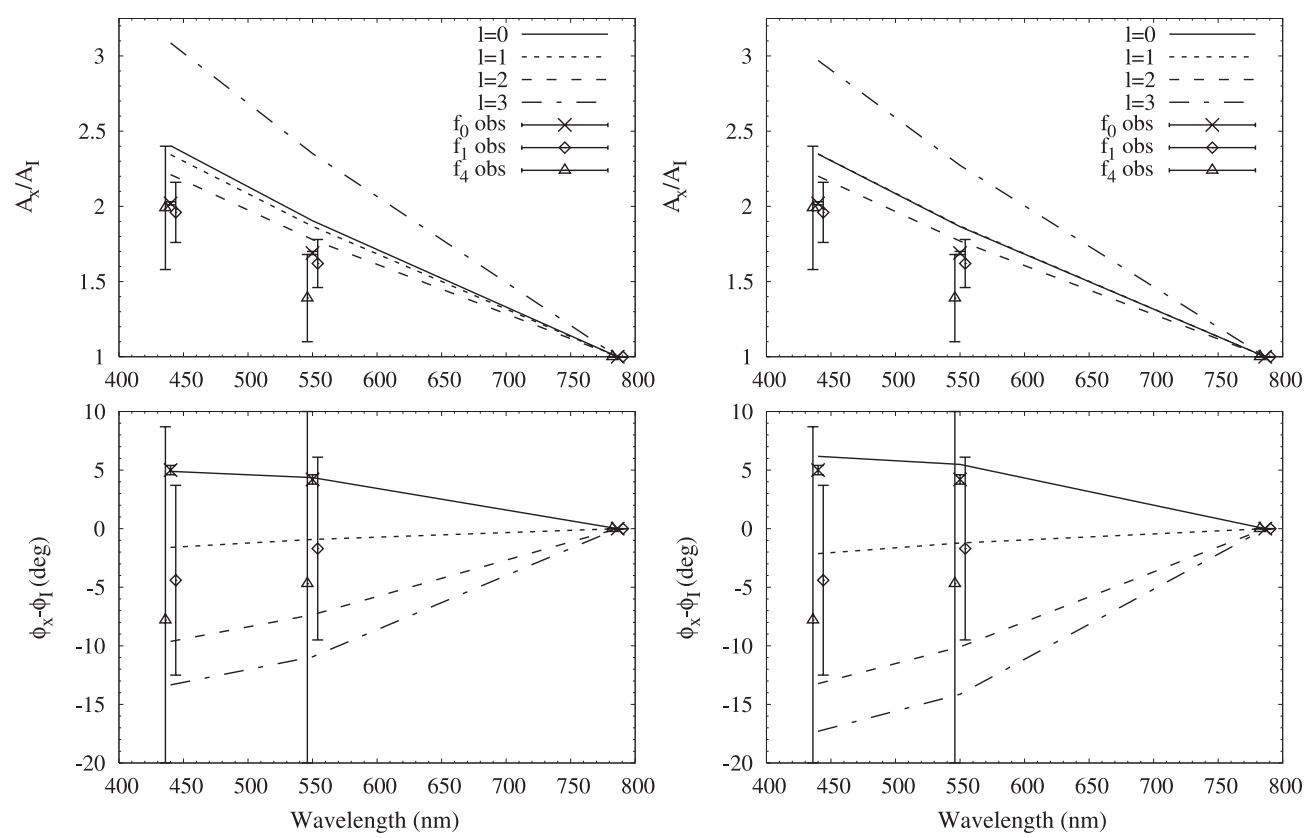

Fig. 7. Theoretical and observed $B / I$ and $V / I$ amplitude ratios (top panels) and phase differences (bottom panels) for a model with $\alpha=1$ (left panels) and $\alpha=1.8$ (right panels). The lines are the theoretical predictions for $\ell=0$ (solid line), $\ell=1$ (dotted line), $\ell=2$ (dashed line) and $\ell=3$ (dot-dashed line). The error bars correspond to the observations for $f_{0}, f_{1}$ and $f_{4}$.

cannot be completely discarded. For a $0.98 M_{\odot}$ model, fitting exactly $f_{0}$ as the radial fundamental mode, the corresponding global parameters are $\log T_{\text {eff }}=3.900, \log L / L_{\odot}=0.674$ and $\log g=4.308$.

Identification of fundamental radial mode for the main frequency of BL Cam is in good agreement with that commonly derived for the main frequency in high amplitude $\delta$ Sct and SX Phe-type variables. This identication was already suggested for BL Cam by earlier authors on the basis of the amplitude and shape of the light curves (McNamara 1995) and the derived value of 0.031 for the pulsation constant $\mathrm{Q}$.

In model $1(\alpha=1.8)$, the range of unstable radial modes predicted by our non-adiabatic code goes from $\mathrm{p}_{1}$ up to $\mathrm{p}_{6}$ (freq. $\left.\sim 60 \mathrm{~cd}^{-1}\right)$; and for model $2(\alpha=1)$, it goes from $\mathrm{p}_{1}$ up to $\mathrm{p}_{5}$ (freq. $\sim 53 \mathrm{~cd}^{-1}$ ). Hence, the excitation of all the observed modes except $f_{21}$ and $f_{22}$ is explained by our models.

\section{Conclusions}

In this work we have used the most reliable contributions of a long and extensive multisite photometric campaign to perform a detailed pulsational study of the extreme metal-deficient field high-amplitude SX Phe-type variable BL Cam. The majority of the secondary modes suspected from earlier works are confirmed here and, additionally, a large number of new peaks are detected. The results show the most complex spectrum ever detected in a high-amplitude pulsator in the Lower Cepheid Instability Strip. It consists of a large number of independent modes with very small amplitudes in addition to the already well-known main periodicity with much larger amplitude.

High-amplitude pulsators in the Lower Cepheid Instability Strip are commonly monoperiodic or double-mode radial pulsators. Only in a very few cases, some additional independent modes have been detected in their light curves which usually are non-radial (Pócs et al. 2002; Poretti 2003, and references therein). In addition to its main periodicity, the dense spectrum of independent modes with small amplitudes found in BL Cam makes this star an exotic object among the high-amplitude pulsators and a unique target for asteroseismological studies. This opens the possibility to find similar microvariability features in other classical high-amplitude objects with the use of better tools: ground-based multisite observations including observations in various passbands and/or very high-quality and homogeneous satellite data.

The radial double-mode nature claimed by some authors for the main two peaks of BL Cam (as fundamental/first overtone or first/second overtone of radial pulsation), typical in multiperiodic high-amplitude $\delta$ Sct and SX Phe-type variables, is not confirmed in this work. As regards the frequency $f_{6}=32.6464 \mathrm{~cd}^{-1}$ could correspond to the first radial overtone, although it is subject to strong amplitude changes with long-term timescale which does not favour this hypothesis. Amplitude variability occurs for the majority of the secondary excited modes, but not for the main periodicity, which seems to remain stable for some decades within the observational uncertainties.

Nature of the main frequency $f_{0}$ as being a radial fundamental mode is confirmed on the basis of multicolour photometry and observed phase-shift and amplitude-ratio for different filters. This agrees well with that commonly occuring in similar high-amplitude pulsators located in the Lower Instability Strip. The secondary peak $f_{1}=25.2523 \mathrm{~cd}^{-1}$ is identified as a mixed nonradial mode $g_{4}$ with $\ell=1$.

Obviously, the full discussion given in this work is on the basis of all the peaks found as significant in our analysis are true independent peaks, corresponding to independent pulsation modes presently excited in BL Cam, with the exceptions of the harmonics $2 f_{0}$ and $3 f_{0}$ and the frequency combination $f_{2}=f_{0}+f_{1}$. Nevertheless, some other arguments can be presented here. It might be that some of the low-amplitude peaks close to the main frequency could be produced by amplitude and/or period variability of $f_{0}$. The presence of a clump of frequencies close to the primary peak, due to the statistical properties of the periodograms, could be indicative of the presence of a peak with a finite width, rather than a multitude of individual frequencies. 
These peaks could therefore be generated by a modulation in amplitude and/or frequency of the main one rather than by multiperiodicity. Even if we accept $f_{1}$ as a real component, such a conclusion could also be applied to it. We have presented arguments that favour the hypothesis of constant amplitude of $f_{0}$ within our observational uncertainties (a few millimagnitudes). However, these uncertainties could be enough to produce the very low-amplitude secondary peaks shown close to $f_{0}$.

On the other hand, Table 3 shows some pairs of close frequencies. In particular, $f_{5}-f_{14}=0.066 \mathrm{~cd}^{-1}$ and $f_{4}-f_{0}=$ $0.062 \mathrm{~cd}^{-1}$. They could indeed be separated frequency pairs or single modes with variable amplitude. The total time span of our observations is of $\Delta T=128$ days. This means a frequency resolution of about $1.5 /(\Delta T)=0.012 \mathrm{~cd}^{-1}$ (Loumos \& Deeming 1978) which seems to be enough to well separate these frequency pairs. Moreover, the frequency $f_{4}$ was already detected by F06 in at least two different data sets.

Concerning the amplitude variability detected in some of the secondary peaks: it might be that some of this variability is produced by additional excited modes too close to the already detected ones to be resolved by our present data set. To gain insights on these questions, better data sets are needed based on new and extensive high-quality multisite observations.

Acknowledgements. E.G.H. acknowledges the use of the 1.8-m Plaskett Telescope at the Dominion Astrophysical Observatory, Herzberg Institute of Astrophysics, National Research Council of Canada. J.H.S. is very grateful to his group of students at the Physics Department of the Virginia Polytechnic Institute and State University. This investigation was partially supported by the Junta de Andalucía, the Spanish Dirección General de Investigación (DGI) under projects AYA2006-06375 and ESP2004-03855-C03-01, and the Grant Agency of the Czech Republic, grant No. 205/06/0217. J.C.S. acknowledges support by an I3P contract partially financed by the European Social Fund. The authors thank an anonymous referee for several valuable comments which helped to improve this paper. Acknowledgements are also made to the staff of the SNO for their help during the observations and Dr. V. Costa for proofreading. The $1.5 \mathrm{~m}$ telescope at SNO is operated by the Instituto de Astrofísica de Andalucía. Part of the data for this research was acquired with equipment purchased thanks to a research fund financed by the Belgian National Lottery (1999). M.H. acknowledges support by the Turkish National Observatory. This research has made use of both the Simbad database, operated at CDS, Strasbourg, France, and the Astrophysics Data System, provided by NASA, USA.

\section{References}

Balona, L. A., \& Evers, E. A. 1999, MNRAS, 302, 349

Breger, M., Stich, J., Garrido, R., et al. 1993, A\&A, 271, 482

Breger, M., Handler, G., Garrido, R., et al. 1999, A\&A, 349, 225

Breger, M., Lenz, P., Antoci, V., et al. 2005, A\&A, 435, 955

Claret, A. 2000, A\&A, 363, 1081

Dupret, M.-A. 2002, Ph.D. Thesis, Bull. Soc. Roy. Sc. Liège, 5-6, 249

Dupret, M.-A., Grigahcène, A., Garrido, R., et al. 2005, MNRAS, 361, 476

Fauvaud, S., Rodríguez, E., Zhou, A.-Y., et al. 2006, A\&A, 451, 999 (F06)

Fauvaud, S., et al. 2007, A\&A, in preparation

Garrido, R., \& Rodríguez, E. 1996, MNRAS, 281, 696

Green, R. B. 1985, Spherical Astronomy (Cambridge University Press)

Grigahcène, A., Dupret, M.-A., Gabriel, M., et al. 2005, A\&A, 434, 1055

Hintz, E. G., Joner, M. D., McNamara, D. H., Nelson, K. A., \& Moody, J. W. 1997, PASP, 109, 15

Kim, C., Jeon, Y. B., \& Kim, S. L. 2003, PASP, 115, 755

Kurucz, R. L. 1993, ATLAS9 Stellar Atmosphere programs and $2 \mathrm{~km} \mathrm{~s}^{-1}$ grids. Kurucz CDROM No. 13

Lenz, P., \& Breger, M. 2005, CoAst, 146, 5

Loumos, G. L., \& Deeming, T. J. 1978, Ap\&SS, 56, 285
McNamara, D. H. 1995, AJ, 109, 1751

McNamara, D. H. 1997, PASP, 109, 1221

Montgomery, M. H., \& O’Donoghue, D. 1999, Delta Scuti Star Newsletter, 13, 28

Moya, A., Garrido, R., \& Dupret, M.-A. 2004, A\&A, 414, 1081

Pócs, M. D., Szeidl, B., \& Viraghalmy, G. 2002, A\&A, 393, 555

Poretti, E. 2003, A\&A, 409, 1031

Poretti, E., Suárez, J. C., Niarchos, P. G., et al. 2005, A\&A, 440, 1097

Rodríguez, E., Rolland, A., López-González, M. J., \& Costa, V. 1998, A\&A, 338,905

Rodríguez, E., López-González, M. J., \& López de Coca, P. 2000, A\&AS, 144, 469

Suárez, J. C., Garrido, R., \& Goupil, M. J. 2006, A\&A, 447, 649

Walraven, T., Walraven, J., \& Balona, L. A. 1992, MNRAS, 254, 59

Zhou, A.-Y., Rodríguez, E., Jiang, S. Y., Rolland, A., \& Costa, V. 1999, MNRAS, 308, 631

Zhou, A.-Y., Du, B. T., Zhang, X. B., \& Zhang, R. X. 2001, IBVS, 5061

1 Instituto de Astrofísica de Andalucía, CSIC, PO Box 3004, 18080 Granada, Spain e-mail: eloy@iaa.es

2 Association AstroQueyras, Le Bois de Bardon, Taponnat, 16110 La Rochefoucauld, France e-mail: stephane. fauvaud@wanadoo.fr

3 Groupe Européen d'Observations Stellaires (GEOS), 23 Parc de Levesville, 28300 Bailleau l'Evêque, France

4 Sulphur Flats Observatory, 449 Sulphur Creek Road, Jemez Springs, NM 87025, USA

5 National Astronomical Observatories, Chinese Academy of Sciences, Beijing 100012 China

6 Observatoire de la Côte d'Azur, BP 4229, 06304 Nice cedex 4, France

7 LESIA, Observatoire de Paris-Meudon, UMR 8109, 92190 Meudon, France

8 CRAAG, Algiers Observatory, BP 63 Bouzareah 16340, Algiers, Algeria

9 Instituut voor Sterrenkunde, Katholieke Universiteit Leuven, Celestijnenlaan 200 B, 3001 Leuven, Belgium

10 Variable Star Section, Norwegian Astronomical Society, PO Box 1029 Blindern, 0315 Oslo, Norway

11 Astronomical Institute, Charles University Prague, V Holesovickach 2, 18000 Praha 8, Czech Republic

12 Beersel Hills Observatory, B-Beersel, Belgium

13 Koninklijke Sterrenwacht van België, Ringlaan 3, 1180 Brussel, Belgium

14 Ankara University, Science Faculty, Department of Astronomy and Space Sciences, 06100 Ankara, Turkey

15 Department of Physics and Astronomy, Brigham Young University, N283 ESC, Provo, UT 84602, USA

16 Observatory and Planetarium of Johann Palisa, VSB-Technical University, Listopadu 15, 70833 Ostrava-Poruba, Czech Republic

17 Martin Observatory, Physics Dep., Virginia Polytechnic Institute \& State Univ., Blacksburg, VA 24061, USA

18 Astronomical Institute, Academy of Sciences, 25165 Ondrejov, Czech Republic

19 Club d'Astronomie Lyon Ampère, 37 rue Paul Cazeneuve, 69008 Lyon, France

20 Club Eclipse, 22 rue du Borrégo, Appt 5A4, 75020 Paris, France

21 Ege University Observatory, 35100 Bornova, Izmir, Turkey

22 American Association of Variable Star Observers, 25 Birch St., Cambridge, MA 02138, USA

23 Akdeniz University, Art \& Science Faculty, Dep. of Physics, Antalya, Turkey 